\title{
ERBIUM DOPED FIBER AMPLIFIER PERFORMANCE USING DIFFERENTHOST MATERIALS IN THE BAND 1450-1650 NM: A COMPARATIVE STUDY
}

\author{
YAHY A M. Zakaryia ${ }^{1}$, Moustafa H. Aly ${ }^{2 *}$, OSAMa MAHran ${ }^{3}$, AhMEd E. El- \\ SAMAHY ${ }^{3}$ \\ ${ }^{1}$ Faculty of Engineering, University of Alexandria, Alexandria, Egypt \\ ${ }^{2}$ Faculty of Engineering, Arab Academy for Science \& Technology, Alexandria, Egypt \\ ${ }^{3}$ Faculty of Science, University of Alexandria, Alexandria, Egypt \\ e-mail:mosaly@aast.edu
}

\begin{abstract}
A numerical investigation of the performance characteristics of erbium doped fiber amplifier using different host materials is presented. The emission and absorption curves of each of these hosts are fitted to Guassian fitting parameters. A software program is then implemented to calculate the gain coefficient, gain spectrum and the equivalent input noise factors in forward and reverse directions. The hosts under consideration are: almino-germanosilicate, bismuth, $\mathrm{LiNbO}_{3}$, tellurite, sodium niobium phosphate, oxyfluoride silicate, $\mathrm{Al}_{2} \mathrm{O}_{3}$ and fluoride phosphate glasses. The corresponding gain covers the $1450-1650 \mathrm{~nm}$ wavelength range.
\end{abstract}

\section{INTRODUCTION}

As an optical signal propagates through a fiber, its power dissipates as a result of absorption and scattering [1]. Optical signals have to be regenerated for longer distance propagation. Electronic repeaters are commonly used to restore the signal. Such repeaters limit the progress in optical communication systems due to their relatively slower electronic speed that hinders the transmission rates of the system. Two decades ago, attention has been diverted toward optical amplifiers because of their advantages over electronic repeaters [2-5]. Optical amplification may be attained by either semiconductor optical amplifiers or fiber amplifiers. The erbium doped fiber amplifier receive more attention because its emission coincides with the $1.55 \mu \mathrm{m}$ window, corresponding to minimum attenuation in silica fibers. The most important parameters of optical amplifiers are their gain spectrum and noise properties. It is important that the maximum gain be centered at the wavelength of interest. The almino-germanosilicate glass exhibits high gain, high saturation output power, polarization independent gain and no crosstalk [6]. While the host, bismuth glass, exhibits broadened and flattened gain spectra of (EDFA), low noise figure and low insertion loss [7]. The host, $\mathrm{LiNbO}_{3}$ glass, is considered as one of the most popular dielectric materials in optoelectronics for remarkable electro-optical, acousto-optical and nonlinear properties [8]. The tellurite based fiber amplifiers and lasers, which can be used for the L-band operation, are capable of providing large and broad stimulated emission cross section because of their high refractive indices at the 
communication band, and are attractive host materials for broadband applications [9]. The host, sodium niobium phosphate glass shows good quality optical waveguides by the ionexchange technique [10]. The oxyfluoride silicate can be used for potential broadband optical amplifier in the wavelength-division-multiplexing (WDM) network system [11]. The use of the host $\mathrm{Al}_{2} \mathrm{O}_{3}$ results in a high mode intensity in the waveguides and allows for a small bending radius and thus compact waveguide devices [12]. Fluoride phosphate glasses are interesting gain materials for lasers and amplifiers in the eye-safe region around $1.5 \mu \mathrm{m}$ for applications in telecommunication systems, medicine and meteorology [13].

\section{THEORY}

\subsection{Rate Equation in Three Level Systems} [6]:

The rate equations corresponding to the specific case of confined Er-doping are

$$
\begin{aligned}
& \pm \frac{d q^{ \pm}}{d z}=\left(\gamma_{e p}-\gamma_{a p}\right) q^{ \pm} \\
& \pm \frac{d p_{k}^{ \pm}}{d z}=\left(\gamma_{e k}-\gamma_{a k}\right) p_{k}^{ \pm}+2 \gamma_{e k} p_{o k}
\end{aligned}
$$

where $q$ is the normalized pump power, $\mathrm{p}_{\mathrm{k}}$ is the normalized sum of the signal power and the noise power introduced by the amplified spontaneous emission (ASE) at a signal wavelength $\lambda_{k}$, the positive sign refers to the forward direction while the negative sign refers to the reverse direction and $p_{o k}$ is the equivalent input noise power normalized to the saturation power at $\lambda_{k}$. The emission and absorption coefficients are $\gamma_{e}, \gamma_{a}$ and the letters $\mathrm{p}$ and $k$ correspond to the pump and the signal, respectively.

For the three-level pumping scheme, the emission and absorption coefficients are defined as [14]:

$$
\begin{gathered}
\gamma_{a p}=\alpha_{p} \frac{1+\sum_{j} \frac{\eta_{k}}{1+\eta_{j}}\left(p_{j}^{+}+p_{j}^{-}\right)}{1+q^{+}+q^{-}+\sum_{j}\left(p_{j}^{+}+p_{j}^{-}\right)} ; \gamma_{e p}=0, \\
\gamma_{e k}=\alpha_{k} \frac{\eta_{k}\left(q^{+}+q^{-}\right)+\sum_{j} \frac{\eta_{k}}{1+\eta_{j}}\left(p_{j}^{+}+p_{j}^{-}\right)}{1+q^{+}+q^{-}+\sum_{j}\left(p_{j}^{+}+p_{j}^{-}\right)},
\end{gathered}
$$




$$
\gamma_{a k}=\alpha_{k} \frac{1+\sum_{j} \frac{\eta_{k}}{1+\eta_{j}}\left(p_{j}^{+}+p_{j}^{-}\right)}{1+q^{+}+q^{-}+\sum_{j}\left(p_{j}^{+}+p_{j}^{-}\right)}
$$

where

$$
\alpha_{p}=\rho_{o} \sigma_{a p} \Gamma_{p}
$$

and

$$
\alpha_{k}=\rho_{o} \sigma_{a k} \Gamma_{k}
$$

where $\alpha_{\mathrm{p}}$ is the pump and $\alpha_{k}$ is the signal absorption coefficients, $\Gamma_{p, k}$ the overlap factors of the pump and signal at $\lambda_{p \mathrm{k}}$, the wavelength of pump and signal and $\rho_{o}$ is the dopant density.

\subsection{Amplifier Gain}

Introducing the emission and absorption coefficients to the power rate equations, one can get:

$$
p_{L}^{+}=p_{o}^{+} \exp \left[\frac{\alpha_{k}}{\alpha_{p}}\left\{\frac{\eta_{p}-\eta_{k}}{1+\eta_{p}}\left(q_{L}^{+}-q_{o}^{+}\right)+\log \left(\frac{q_{L}^{+}}{q_{o}^{+}}\right)\right\}\right]
$$

where $p_{o}^{+}$and $p_{L}^{+}$are the normalized input and output signal powers, $\eta_{p}$ and $\eta_{k}$ are the ratio of the emission to the absorption cross section for the pump and the signal, respectively.

From Eq.(8), the amplifier gain, $G$, can be obtained as:

$$
G=\frac{p_{L}^{+}}{p_{o}^{+}}=\exp \left[\frac{\alpha_{k}}{\alpha_{p}}\left\{\frac{\eta_{k}-\eta_{p}}{1+\eta_{p}}\left(q_{L}^{+}-q_{o}^{+}\right)+\log \left(\frac{q_{L}^{+}}{q_{o}^{+}}\right)\right\}\right]
$$

From the definition of the absorption coefficient, $\alpha_{p}$, and using an amplifier of length $L$, one can get the relation between the input and output pump powers, $q_{o}$ and $q_{L}$, as:

$$
q_{o}^{+}-q_{L}^{+}=\alpha_{p} L-\log \left(\frac{q_{o}^{+}}{q_{L}^{+}}\right) .
$$

Equation (9) is now used with Eq.(10) to get:

$$
q_{L}^{+}=q_{o}^{+} \exp \left(-A_{G} L\right)
$$

where $A_{G}$ is the gain-dependant pump absorption coefficient, given by: 


$$
A_{G}=\alpha_{p}\left(\frac{1+\eta_{p}}{1+\eta_{k}}\right)\left(\frac{\eta_{k}-\eta_{p}}{1+\eta_{p}}-\frac{\log G}{\alpha_{k} L}\right)
$$

The input pump power $q_{o}^{+}$is then expressed as a function of the amplifier gain $G$, by eliminating $q_{L}^{+}$from Eqs.(11) and (12) as:

$$
q_{o}^{+}=\alpha_{p} L \frac{Q_{k}}{1-\exp \left(-\alpha_{p} L\left(1-Q_{k}\right)\right)}
$$

with

$$
Q_{k}=\frac{1+\eta_{p}}{1+\eta_{k}}\left(1+\frac{\log (G)}{\alpha_{k} L}\right)
$$

The output pump power $q_{L}^{+}$is then rewritten:

$$
q_{L}^{+}=q_{o}^{+} \exp \left(-\alpha_{p} L\left(1-Q_{k}\right)\right)
$$

For forward pumping case, $q_{L}^{+}$is always smaller than $q_{o}^{+}$and hence $Q_{k}$ must be smaller than one. Equation (14) gives the following condition for the peak gain as:

$$
G<\exp \left(\frac{\eta_{k}-\eta_{p}}{1+\eta_{p}} \alpha_{k} L\right)
$$

which sets the upper limit for the gain, $G_{\max }$, through which the amplifier length, $L$, can be determined.

Through the amplifier gain, one can get the normalized input and output signal powers as:

$$
p_{L}^{+}=G_{k}^{+} p_{o}^{+}+\left(2 p_{o k}\right) N^{+}
$$

and

$$
p_{o}^{-}=G_{k}^{-} p_{L}^{-}+\left(2 p_{o k}\right) N^{-}
$$

where $N^{+}$and $N^{-}$are the ASE photon numbers in forward and backward directions, respectively, which are given by:

$$
N^{+}\left(\lambda_{k}\right)=\left(\frac{\alpha_{k}}{\alpha_{p}}\right)\left(\frac{\eta_{k}}{1+\eta_{p}} I^{+}\left(q_{o}, q_{L}\right)\right) \times \exp \left[\left(\frac{\alpha_{k}}{\alpha_{p}}\right)\left(\log \left(q_{L}\right)-\frac{\eta_{k}-\eta_{p}}{1+\eta_{p}} q_{L}\right)\right]
$$




$$
N^{-}\left(\lambda_{k}\right)=\left(\frac{\alpha_{k}}{\alpha_{p}}\right)\left(\frac{\eta_{k}}{1+\eta_{p}} I^{-}\left(q_{o}, q_{L}\right)\right) \times \exp \left[\left(-\frac{\alpha_{k}}{\alpha_{p}}\right)\left(\log \left(q_{o}\right)-\frac{\eta_{k}-\eta_{p}}{1+\eta_{p}} q_{o}\right)\right],
$$

with

$$
I^{ \pm}\left(q_{o}, q_{L}\right)=\int_{q_{L}}^{q_{o}} \frac{\exp \left( \pm \frac{\alpha_{k}}{\alpha_{p}} \frac{\eta_{k}-\eta_{p}}{1+\eta_{p}}\right)}{X^{ \pm \alpha_{s} l \alpha_{p}}} d X
$$

\subsection{Equivalent Input Noise Factor}

In terms of the gain and the ASE noise photon numbers, the equivalent input noise factors for the forward and backward pumping schemes, $n_{e q}^{ \pm}$, and the optical noise figures, $F_{o}^{ \pm}$, are defined by [14]:

$$
\begin{aligned}
& n_{e q}^{ \pm}\left(\lambda_{k}\right)=\frac{N^{ \pm}\left(\lambda_{k}\right)}{G}, \\
& F_{o}^{ \pm}(\lambda)=\frac{1+2 N^{ \pm}\left(\lambda_{k}\right)}{G} .
\end{aligned}
$$

Using Eqs. (19)-(21), the equivalent noise factors and the optical noise figures can be written in the forms:

$$
\begin{aligned}
& n_{e q}^{ \pm}\left(\lambda_{k}\right)=\left(\frac{\alpha_{k}}{\alpha_{p}}\right)\left(\frac{\eta_{k}}{1+\eta_{p}} I^{ \pm}\left(q_{o}, q_{L}\right)\right) \exp \left[\left(\frac{\alpha_{k}}{\alpha_{p}}\right) \log \left(q_{o}\right)-\frac{\eta_{k}-\eta_{p}}{1+\eta_{p}} q_{o}\right] \\
& F_{o}^{ \pm}\left(\lambda_{k}\right)=2 n_{e q}^{ \pm}\left(\lambda_{k}\right)+\frac{1}{G}
\end{aligned}
$$

\section{RESULTS}

The experimental emission and absorption cross sections of the different hosts [3-16] are fitted to Gaussian curves and the parameters are used in calculations of the gain coefficient, gain spectrum and the equivalent input noise factors in forward and reverse directions. One considers an EDFA pumped at $\lambda_{p}=980 \mathrm{~nm}$, at which the EDFA operates as a three-level laser system, with the ratio of emission to absorption cross section of pump $\eta_{\mathrm{p}}=0$.

Figure 1 plots the gain coefficient around the $1.5 \mu \mathrm{m}$ for a typical $\mathrm{Er}^{3+}$-doped aluminogermanosilicate glass fiber amplifier for different values of the relative inversion $\mathrm{D}$, by 
incremental steps of 0.2. It is clear that for $\mathrm{D}=-1$, all ions are in the ground state and the medium is absorbing at all signal wavelengths, as the gain coefficient is negative. As the value of $\mathrm{D}$ increases, however, a spectral region near the long wavelength side of the transition is characterized by a positive gain coefficient.

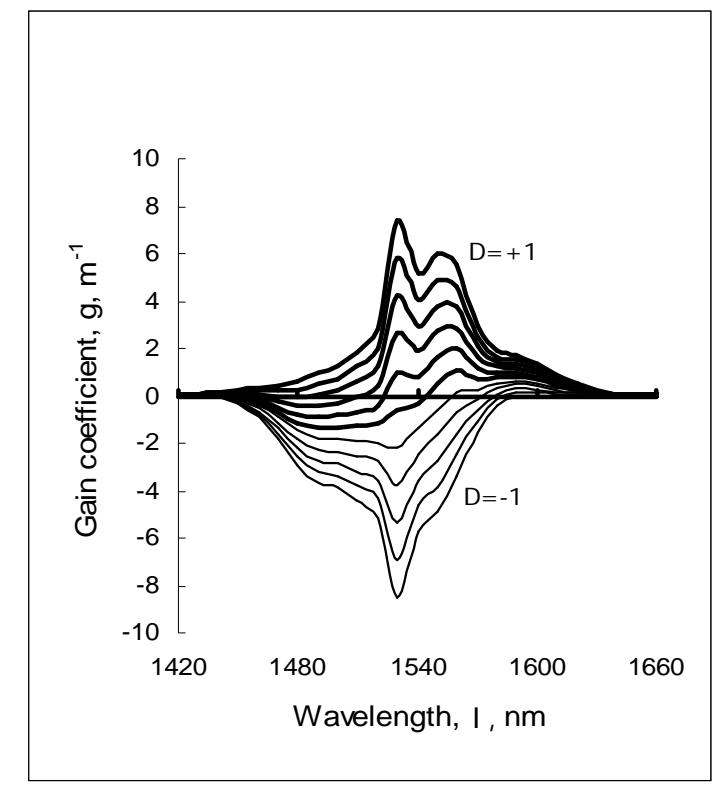

Fig.1: Signal gain coefficient of erbium doped alumino-germanosilicate glass.

The gain coefficient for the hosts, bismuth glass, $\mathrm{LiNbO}_{3}$ glass, tellurite, sodium niobium phosphate glass, oxyfluoride silicate, $\mathrm{Al}_{2} \mathrm{O}_{3}$ and fluoride phosphate glass fiber amplifier, is calculated in the same manner. The EDFA gain spectrum $G(\lambda)$ corresponding to the hosts, alumino-germanosilicate glass, bismuth glass, $\mathrm{LiNbO}_{3}$ glass and tellurite is shown in Fig.2, while that of the sodium niobium phosphate glass, oxyfluoride silicate, $\mathrm{Al}_{2} \mathrm{O}_{3}$ and fluoride phosphate glass hosts is shown in Fig.3.

Figure 4 displays the dependence of the equivalent noise figure on both signal gain and signal wavelength for the forward pumping for the alumino-germanosilicate glass at different values of the maximum gain. It is noted that, as the gain increases the equivalent input noise figure becomes nearly independent of signal wavelength and for the case of complete population inversion it reaches unity. Such a regime can be achieved with a pump wavelength of $980 \mathrm{~nm}$, for which the EDFA operates as a three-level system, as mentioned before. 

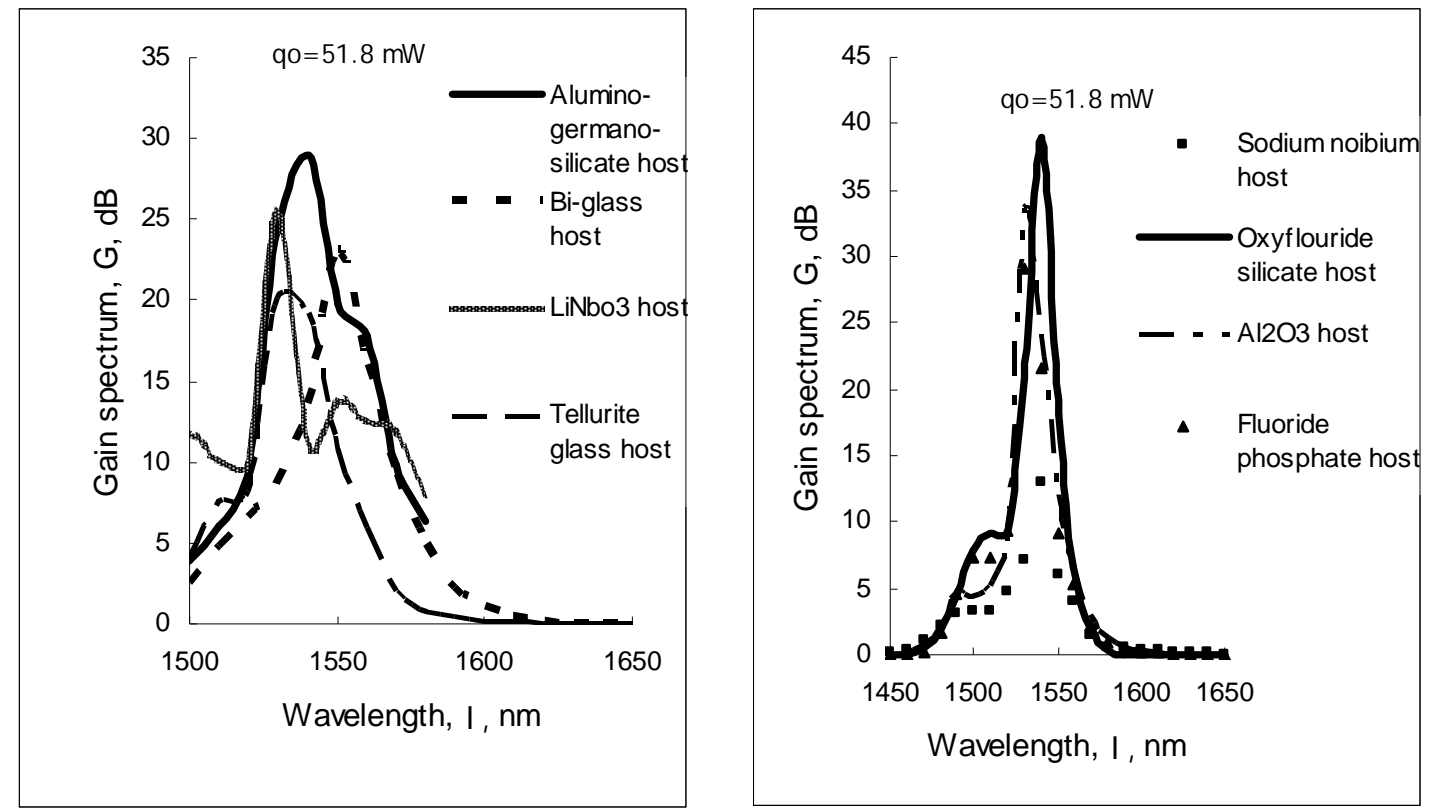

Fig. 2: Gain spectrum plotted at $q_{o}=51.8 \mathrm{~mW}$. Fig. 3: Gain spectrum plotted at $q_{o}=51.8 \mathrm{~mW}$.

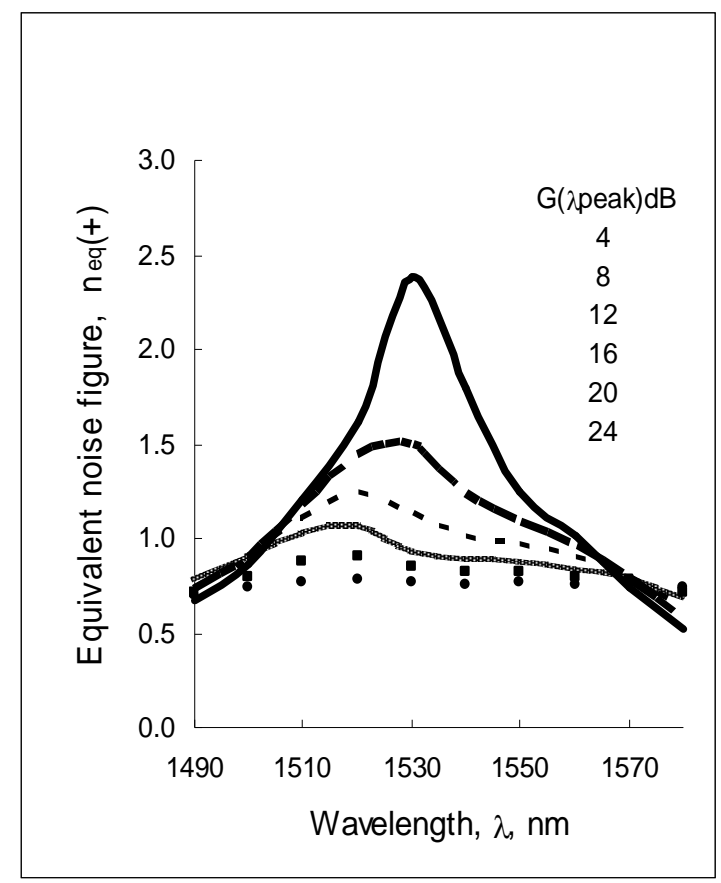

Fig.4.Forward equivalent noise figure for the erbium doped alumino-germanosilicate 
Figures 5 and 6 display the dependence of the equivalent noise figure on both input normalized pump power and signal wavelength for the forward pumping, for the eight mentioned hosts, at the maximum gain of each host.

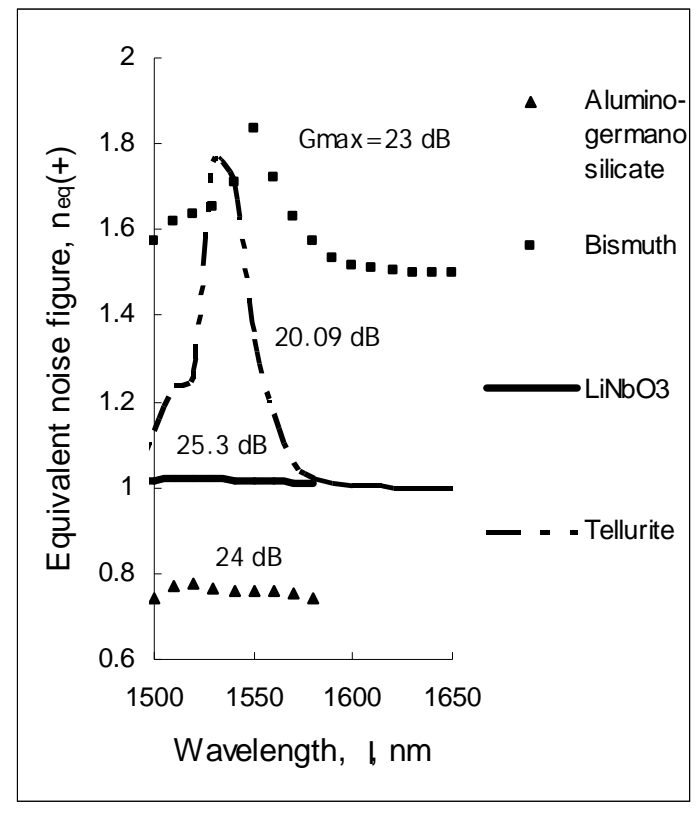

Fig. 5: Forward equivalent noise figure for the erbium doped in aluminogermanosilicate, bismuth, $\mathrm{LiNbO}_{3}$ and tellurite glasses, respectively.

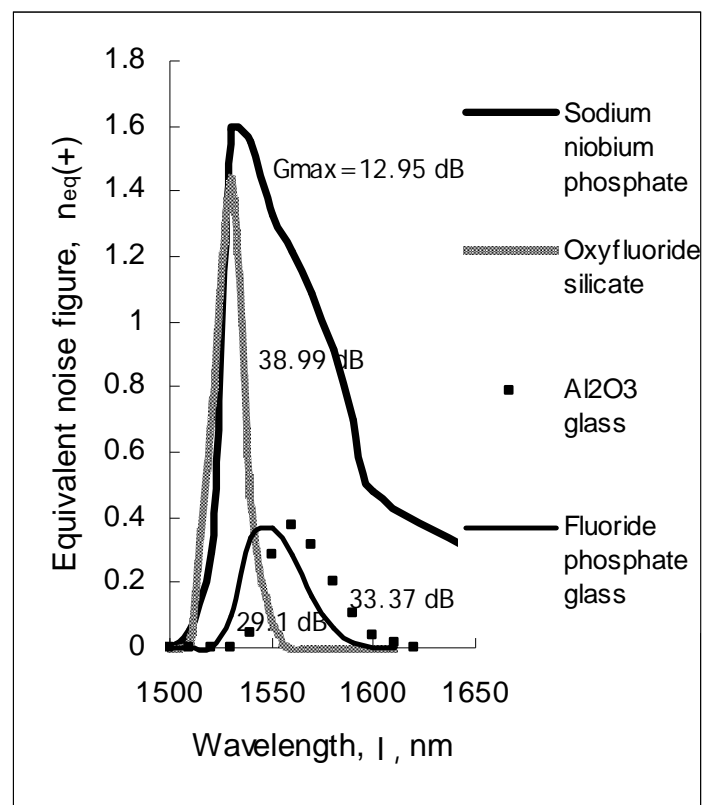

Fig. 6: Forward equivalent noise figure for the erbium doped in, sodium niobium phosphate, oxyfluoride silicate, $\mathrm{Al}_{2} \mathrm{O}_{3}$ and fluoride phosphate glasses, respectively.

The optical noise figure for the almino-germanosilicate glass fiber amplifier is plotted in Figs. 7, at different values of the signal gain G, having its maximum value around 1530 nm.

The optical noise figure for the almino-germanosilicate, bismuth, $\mathrm{LiNbO}_{3}$, tellurite, sodium niobium phosphate, oxyfluoride silicate, $\mathrm{Al}_{2} \mathrm{O}_{3}$ and fluoride phosphate glass fiber amplifiers is plotted in Figs. 8 and 9 at the maximum gain of each host. Figures 7 and 8 indicate that for the highest gains, the optical noise figure reaches a lower limit of $3 \mathrm{~dB}$ and is very nearly wavelength independent.

For the un-pumped fiber $\left(\mathrm{q}_{\mathrm{o}}=0\right)$, the optical noise figure is the reciprocal of the fiber transmission. As the fiber is pumped with an increasing input power, corresponding to increasing gains and overall fiber inversion, the optical noise figure is seen to decrease for forward propagation direction. 


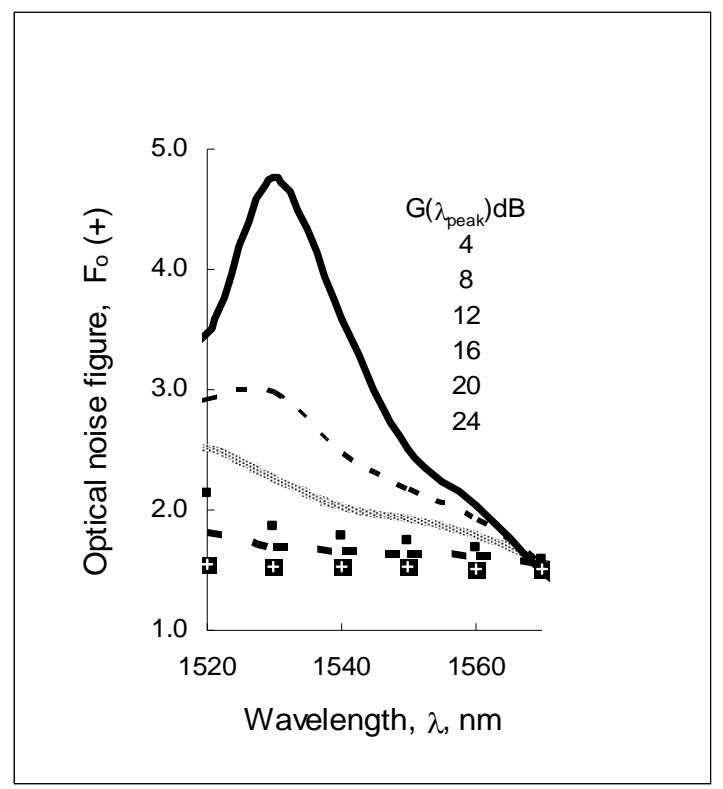

Fig.7: Forward optical noise figure for the erbium doped alumino germanosilicate glass.

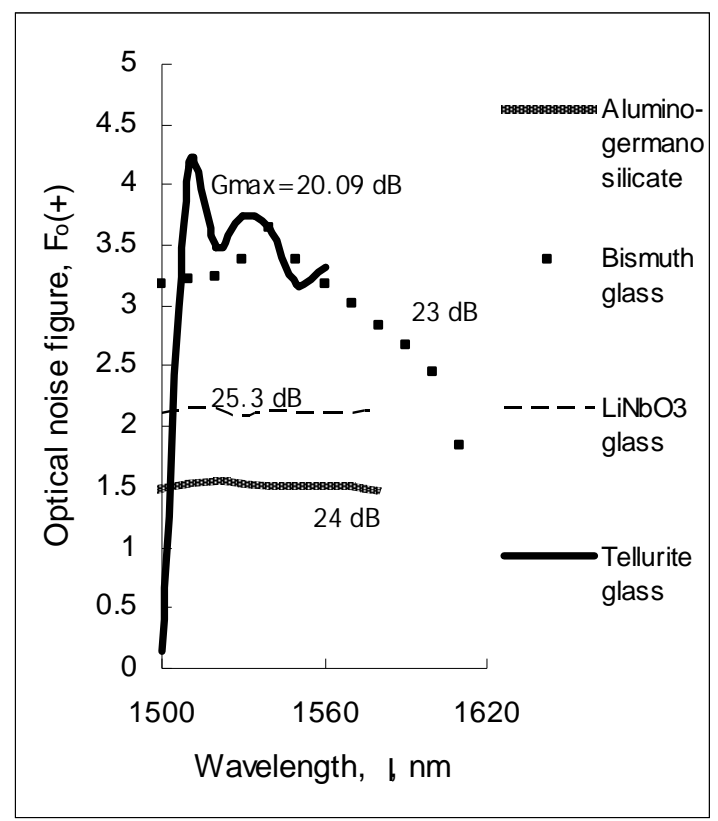

Fig .8: Forward optical noise figure for the erbium doped in, alumino-germanosilicate, bismuth, $\mathrm{LiNbO}_{3}$ and tellurite glasses, respectively.erbium doped with the hosts, alumino germanosilicate glass, bismuth glass, $\mathrm{LiNbO}_{3}$ glass and tellurite respectively.

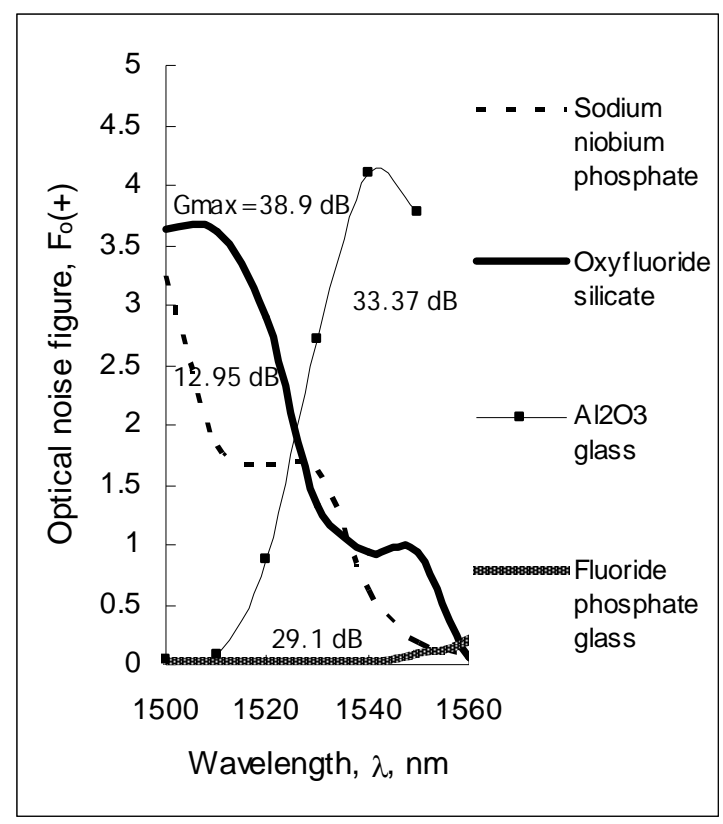

Fig. 9: Forward optical noise figure for the erbium doped in, sodium niobium phosphate, oxyfluoride silicate, $\mathrm{Al}_{2} \mathrm{O}_{3}$ and fluoride phosphate glasses, respectively. 


\section{CONCLUSION}

A numerical investigation of the performance characteristics of erbium-doped in the host materials, almino-germanosilicate, bismuth, $\mathrm{LiNbO}_{3}$, tellurite, sodium niobium phosphate, oxyfluoride silicate, $\mathrm{Al}_{2} \mathrm{O}_{3}$ and fluoride phosphate glass fiber amplifiers. One summarize the features of the amplifier with different hosts in Table 1 , where $G$ is the amplifier gain, $\Delta \lambda$ is the $3 \mathrm{~dB}$ broadening of the gain, $F_{0}$, is the noise figure calculated at the maximum gain, $G_{\max }$, and $\lambda_{0}$ is the central frequency which corresponds to the maximum gain. The amplifier gain using these different hosts covers the wavelength range $1450-1650 \mathrm{~nm}$.

From Table 1, it is clear that the erbium-doped oxyfluoride silicate fiber amplifier exhibits a maximum gain of $39 \mathrm{~dB}$ at the central wavelength of $1540 \mathrm{~nm}$ and minimum noise figure $\leq 1 \mathrm{~dB}$, but the broadening in the gain curve is just $21 \mathrm{~nm}$. One can also see that the erbium doped alumino-germanosilicate fiber amplifier exhibits a more broadening in the gain curve $(=40 \mathrm{~nm})$, which allows amplifying the signal at a large range of wavelengths, which is the case in WDM systems.

The case of erbium doped sodium niobium phosphate glass fiber amplifier exhibits a less gain $(=12.9 \mathrm{~dB})$ than other hosts and large noise figure $(=4 \mathrm{~dB})$ in comparison with the other hosts, and the broadening $(\Delta \lambda=20 \mathrm{~nm})$ is considerably small.

Table 1 Features of the EDFA with different hosts.

\begin{tabular}{ccccc}
\hline Host & $\lambda_{\mathrm{o}}(\mathrm{nm})$ & $\mathrm{G}_{\max }(\mathrm{dB})$ & $\mathrm{F}_{\mathrm{o}}(\mathrm{dB})$ & $\Delta \lambda(\mathrm{nm})$ \\
\hline Alumino-germanosilicate & 1540 & 24 & 1.5 & 40 \\
Bismuth & 1550 & 23 & 3 & 30 \\
LiNbO ${ }_{3}$ & 1553 & 25.3 & 2.2 & 15 \\
Tellurite & 1530 & 20 & 4 & 28 \\
Sodium niobium phosphate & 1540 & 12.9 & 2 & 21 \\
Oxyfluoride silicate & 1540 & 39 & 1 & 22 \\
$\mathrm{Al}_{2} \mathrm{O}_{3}$ & 1530 & 33.37 & 3.5 & 23 \\
\hline Fluoride phosphate & 1530 & 29 & 0.1 & \\
\hline
\end{tabular}




\section{REFERENCES}

[1] William L. Barnes, Richard I. Laming, Eleanor J. Tarbox, and P. R. Morkel, "Absorption and Emission Cross Section of $\mathrm{Er}^{3+}$ Doped Silica Fibers," IEEE J. Quantum Electron., vol. 27, pp1004-1010, 1997.

[2] M. V. D. Vermelho, Ulf Peschel. and J. stewart Aitchison, "Simple and Accurate Procedure for Modeling Erbium-Doped Waveguide Amplifiers With High Concentration," J. Lightwave Technol., vol. 18, pp 401-408, 2000.

[3] Piotr Myslinki, Dung Nguyen and Jacek Chrostowski, "Effects of Concentration on the Performance of Eribum-Doped Fiber Amplifiers," J. Lightwave Technol., vol. 15, pp112-120, 1997.

[4] Paul Wysocki and Vincent Mazurczyk, "Polarization Dependent Gain in Erbium-Doped Fiber Amplifiers: Computer Model and Approximate Formulas," J. Lightwave Technol., vol. 14, pp 572-583, 1996.

[5] Emmanuel Desurvire and Jay R. Simpson, "Amplification of Spontaneous Emission in Erbium-Doped Single-Mode Fibers," J. Lightwave Technol., vol. 7, pp 835-845, 1989.

[6] E. Desurvire, J. L. Zyskind and J. R. Simpson, "Spectral Gain hole-Burning at $1.53 \mu \mathrm{m}$ in Erbium-Doped Fiber Amplifiers," IEEE Photonics Technol. Lett. vol. 2, pp 246-248, 1990.

[7] Jianhu Yang, Shixun Dai, Yuefen Zhou, Lei Wen, Lili Hu and Zhonghong jiang, "Spectroscopic properties and thermal stability of erbium-doped bismuth-based glass for optical amplifier," J. Appl. Physics, vol. 93, pp 977-983, 2003.

[8] C.H. Huang, L. McCaughan and D. M. Gill, "Evaluation of absorption and emission cross sections of Er-doped $\mathrm{LiNbO}_{3}$, for application to integrated optic amplifiers," J. Lightwave Technol., vol. 12, pp. 803-809, 1994.

[9] X. Peng, F. Song, S. Jiang, N. Peyghambarian, Makoto Kuwata-Gonokami and Lei Xu, "Fiber-taper-coupled L-band $\mathrm{Er}^{3+}$-doped tellurite glass microshere laser," Appl. Phys. Lett., vol. 82, pp. 1497-1499, 2003.

[10] G.C. Righini, S. Pelli, M. Fossi, M. Brenci, A.A. Lipovskii, E.V. Kolobkova, A. Speghini and M. Bettinelli, "Characterization of Er-doped sodium-niobium phosphate glasses," Proceedings of SPIE, vol. 4282, pp. 210-215, 2001.

[11] Shiqing Xu, Zhongmin Yang, Shixun Dai, Jianhu Yang, Lili Hu and Zhonghong Jiang, "Spectral properties and thermal stability of $\mathrm{Er}^{3+}$-doped oxyfluoride silicate glasses for broadband optical amplifier," J. Alloys and Compounds, vol. 4, pp. 142-148, 2003.

[12] Gerlas N.van den Hoven, Jan A. van der Elsken, Albert Polman, Cor van Dam, Koos W. M. Van Uffelen and Meint K. Smit, "Absorption and emission cross section of $\mathrm{Er}^{3+}$ in $\mathrm{Al}_{2} \mathrm{O}_{3}$ waveguides", Appl. Opt. , vol. 36, pp. 3338-3341, 1997.

[13] J.F. Philipps, T. Töpfer, H. Ebendorff-Heidepriem and D. Ehrt, R. Sauerbrey, "Spectroscopic and lasing properties of $\mathrm{Er}^{3+}: \mathrm{Yb}^{3+}$-doped fluoride phosphate glasses," Appl. Phys. B 72, pp.399-405 (2001).

[14] E. Desurvire, Erbium Doped Fiber Amplifiers: Principles and Applications, New York: Wiley, 1994.

[15] Martin Achtenhagen, Robert James Beeson, Feng Pan, Bruce Nyman and Amos Hardy,"Gain and Noise in Ytterbium-Sensitized Erbium-Doped Fiber Amplifiers: Measurements and Simulations," J. Lightwave Technol., vol. 19, pp 1521-1526, (2001). 
[16] Federico Caccavale, Francesco Segato and Ibrahim Mansour, "A Numerical Study of Erbium Doped Active $\mathrm{LiNbO}_{3}$ Waveguides by the Beam Propagation Method," J. Lightwave Technol., vol. 15, pp 2294-2300, 1997.

\section{BIOGRAPHIES}

Yahya Mounir Zakaria was born in 1963 in Alexandria, Egypt. He obtained his B.Sc. in Nuclear Engineering, and M.Sc. in Engineering Physics from Faculty of Engineering Alexandria University in 1986 and 1989, respectively. He obtained his Ph.D. in 1996 from McMaster University, Canada. Currently, he is Assistant Professor at the Engineering Physics Department, Faculty of Engineering, University of Alexandria, Alexandria, Egypt.

Moustahfa Hussein Aly born in 1953 in Alexandria, B.Sc. Electrical Engineering 1976, M.Sc. 1983 and Ph.D. 1987, all from Faculty of Engineering, Alexandria University. Currently, he is a Professor of Communications, College of Engineering and Technology, Arab Academy for Science \& Technology \& Maritime Transport, Alexandria, Egypt

Ahmed Azz El-Arab El-Samay was born 1944 in Alexandria, Egypt. He obtained his B.Sc. and M.Sc. in Physics from Faculty of Science, Alexandria University in 1966 and 1971, respectively.. He obtained his Ph.D. in 1976 from Moscow University, Russia. Currently, he is a Professor, at the Physics Department, Faculty of Science, University of Alexandria, Alexandria, Egypt

Ossama S. Mahran was born in 1970 in Alexandria, Egypt. He obtained his B.Sc., M.Sc., Ph.D. in Physics from Faculty of Science, Alexandria University in 1992, 1999 and 2004, respectively. His current position is Assistant Professor at the Department of Physics, Faculty of Science, University of Alexandria, Alexandria, Egypt. 